\title{
Starspot trek: The motion picture
}

\author{
B. Debski \\ Astronomical Observatory of the Jagiellonian University, Orla 171, 30-244 \\ Krakow, Poland (E-mail: bartlomiej.debski@uj.edu.pl)
}

Received: November 18, 2019; Accepted: December 18, 2019

\begin{abstract}
The longitudinal spot migration can be traced with the observation of the O'Connell effect and maxima separation variations. Judging by the correlations between the light curve parameters, the migration processes are confined to the polar regions of the more massive star in a contact binary.
\end{abstract}

Key words: binaries: close - stars: activity - starspots

\section{Introduction}

Light curves of W UMa-type contact binaries are known to be prone to asymmetries. The most often reported distortion is the uneven levels of the brightness maxima (the so-called $O^{\prime}$ Connell effect). Such a distortion is usually explained with a presence of a starspot on the binary surface. The origin of spots is unclear, but since the work of Mullan (1975), the general approach is that they might be magnetically-driven cool starspots. Therefore, the asymmetry coming from the existence of a subluminous region affects a larger part of the light curve, not just one of the extremum at a time. Moreover, it can vary in time, making the whole phased light curve "shake." This wobbling, or intrinsic variability, of a light curve can be studied using long-time base photometry (Debski et al., 2015). Assuming the light curve intrinsic variability comes from the changes of the starspot itself, it is possible to trace the variations of the spot parameters using, e.g. the standard approach of numerically modeling with the employment of the Wilson-Devinney code (Debski et al., 2014). This approach is focused on the longitudinal spot migration, with little consideration to the spot latitude, temperature, or size. Lacking the ability to pinpoint the spot latitude, it is usually modeled on the stellar equator. This numerical modeling method also requires a considerable computational time and resources, and some input parameters describing the system itself are needed.

\section{Tracing of the spot longitudal migration}

An alternative method of tracing the longitudinal spot migration can be adopted by analyzing the evolution of the O'Connell effect. If we assume that there is only one dominating spot, which is circular, cool and located on the primary 

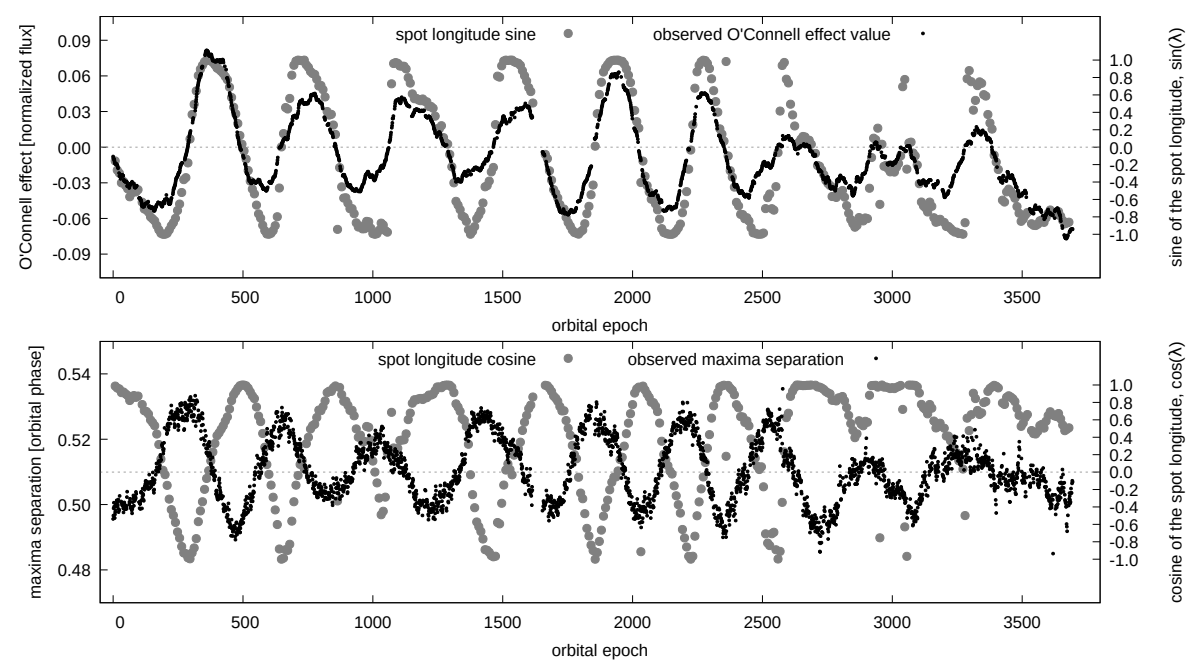

Figure 1. Comparison of the spot longitude found with the numerical modeling methods and the O'Connell effect (top panel) and the maxima separation variations (bottom panel).

component, then the longitude of a spot center would be $\lambda=270^{\circ}$, if the O'Connell effect would be maximally negative (i.e the brightness maximum at orbital phase $\phi=0.25$ would be at its lowest, with respect to the brightness maximum at $\phi=0.75)$. Subsequently, the spot would be centered at the longitude $\lambda=90^{\circ}$ when the O'Connell effect would be maximally positive. The relation between the spot longitude and the O'Connell effect in case of a contact binary KIC 6118779 is shown in the top panel of Fig. 1. The longitude of the spot comes from the numerical modeling process presented in Debski et al. (2014), while the O'Connell effect has been measured with the improved version of the code used in Debski et al. (2015). The spot location is expressed with the sine of the longitude for a better visual aid. The most noticeable fact is that the spot longitude sine is always positive when the O'Connell effect is positive (and vice versa). The numerically-determined spot longitude after epoch $\approx 2500$ is rather chaotic, despite the continued variations of the O'Connell effect. This means that the single-spot longitudinal migration can be traced numerically only if the 'value' of the O'Connell effect varies more or less symmetrically around zero. Tracing the spot migration with the analysis of the O'Connell effect bears no such burdens.

Another light curve parameter that can be used to study the spot migration is the amount of separation between the brightness maxima. As it turns out, this parameter is perfectly anticorrelated with the cosine of the spot longitude (see the bottom panel of Fig. 1). Here, the maxima separation reaches its greatest 

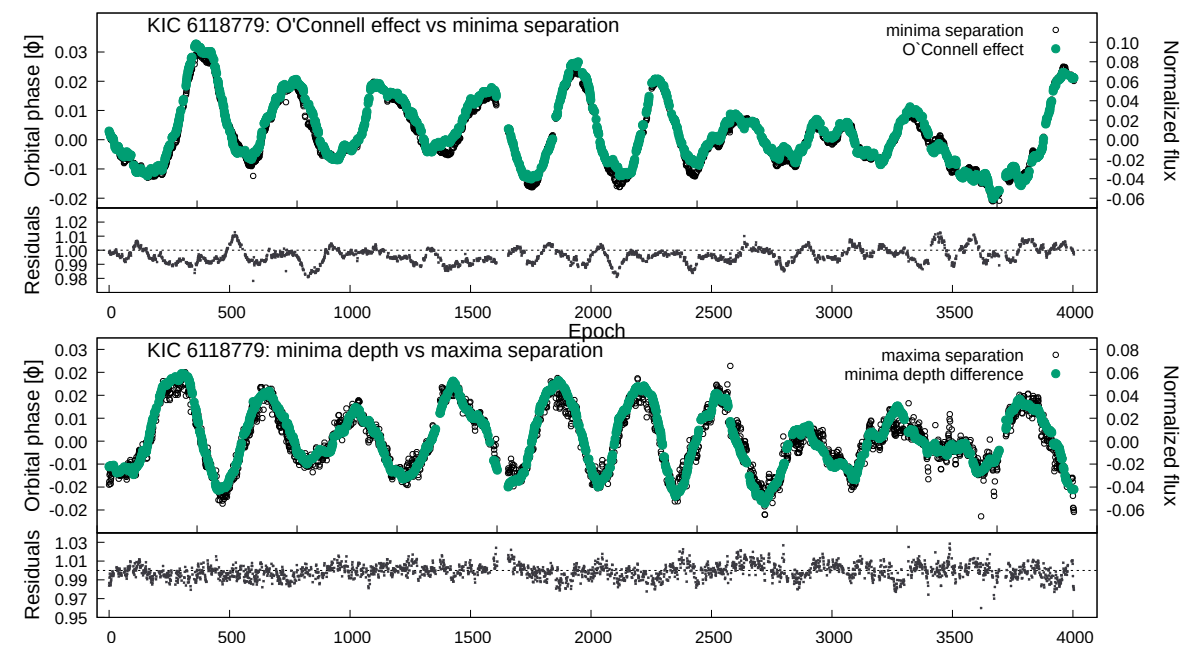

Figure 2. Comparison of the spot longitude found with the numerical modeling methods and the O'Connell effect (top panel) and the maxima separation variations (bottom panel).

value when the dark spot resides on the longitude $\lambda 180^{\circ}$, i.e. lies in the back of a primary component. Alternatively, the maxima are closest to each other when the spot is at $\lambda=0^{\circ}$. Combining the analysis of the maxima separation variations and the evolution of the O'Connell effect leads to the information about the direction of the spot migration. In case of KIC 6118779 the dark spot moves in descending longitudes. The preliminary analysis of contact binaries observed with the Kepler spacecraft (Borucki et al., 2010) results in a conclusion that all studied systems experience descending-longitude spot migration.

It might be interesting to point out a following fine detail in the spot longitude - maxima separation relation. The cosine on the spot longitude is not always negative, when the maxima separation is less than its the median value (marked with a dashed line in the bottom panel of Fig. 1). Instead, the longitude cosine changes its sign always when the maxima separation crosses a value slightly larger (about $0.517 \phi$ instead of the median $0.50994 \phi$ ). This observation might lead to further interesting results when confronted with the analysis of the median maxima separation relation with the mass ratio for contact binaries (Debski, 2019).

\section{Migration latitude and spot size}

In addition to the two above light curve parameters, one can measure the equivalents in their extrema counterparts. The next two light curve parameters are 
therefore the minima depth difference and the separation of the brightness minima. It is most interesting that the variations of the O'Connell effect are the same as the variations of the separation of the brightness minima (with a scaling factor applied). The same situation happens for the evolution of the maxima separation and the minima depth difference variations. This high correlation between light curve parameters is shown in the Fig. 2. Using the means of the numerical simulations, we have established that such occurrence may happen only if the migrating spot is located very close to the stellar pole and must be rather large (radius of a spot reaches about $r=40^{\circ}$ ).

\section{Conclusions}

The longitudinal motion of a spot on a contact binary can be traced with the analysis of the O'Connell effect and the maxima separation variations. Since the light curve parameters vary in a highly correlated fashion, the spot is confined to the polar regions of the more massive component of the binary. That, combined with the fact that the spot must be considerably large, produces a picture of a tilted, precessing polar 'cap.' Our preliminary analysis of the contact binaries observed within the Kepler mission shows that systems with edge-on inclinations nearly always experience a light curve intrinsic variation lead by at least double modulation. At the same time, systems with a very low inclination tend to exhibit just a single-spot caused light curve variations. It is therefore very tempting to end with a conclusion that the spot migration in contact binaries occurs in a form of a precession of large polar spots residing on both poles of the more massive component. That, in turn, opens new possibilities for studying the properties and behavior of the global magnetic field structure in these exotic systems.

Acknowledgements. This work has been supported by the Preludium 12 Grant no. 2016/23/N/ST9/01218 of the Polish National Science Centre.

\section{References}

Borucki, W. J., Koch, D., Basri, G., et al., Kepler Planet-Detection Mission: Introduction and First Results. 2010, Science, 327, 977, DOI: 10.1126/science.1185402

Debski, B., A relation between the brightness maxima separation and mass ratio in contact binaries. 2019, Contributions of the Astronomical Observatory Skalnate Pleso, 49, 258

Debski, B., Baran, A., \& Zola, S., A study of a spot migration in two contact binaries: KIC 2159783 and KIC 6118779. 2014, Contributions of the Astronomical Observatory Skalnate Pleso, 43, 427 
Debski, B., Zola, S., \& Baran, A. 2015, Astronomical Society of the Pacific Conference Series, Vol. 496, Light Curve Morphology: The Evolution of the O'Connell Effect and the Maxima Separation, ed. S. M. Rucinski, G. Torres, \& M. Zejda, 293

Mullan, D. J., On the possibility of magnetic starspots on the primary components of W Ursae Majoris type binaries. 1975, ApJ, 198, 563, DOI: 10.1086/153635 